Adam M. Deane Gordon H. Guyatt

\section{Primum non nocere and challenging conventional treatment}

Received: 26 March 2015

Accepted: 27 March 2015

Published online: 14 April 2015

(C) Springer-Verlag Berlin Heidelberg and ESICM 2015

A. M. Deane (『)

Discipline of Acute Care Medicine, University of Adelaide, Adelaide, SA, Australia

e-mail: adam.deane@adelaide.edu.au

\section{A. M. Deane}

Department of Critical Care Services, Royal Adelaide Hospital, Adelaide, SA, Australia

\section{G. H. Guyatt}

Departments of Clinical Epidemiology and Biostatistics and of Medicine, Faculty of Health Sciences, McMaster University, Hamilton, ON, Canada

If you knew that, for every 80 patients to whom you prescribed an acid-suppressive drug, 1 patient would develop a ventilator-associated pneumonia, and that, for every 100 patients to whom you prescribed these drugs, 1 patient would develop a Clostridium difficile infection, would you continue to prescribe these drugs? What if you knew that you needed to prescribe acid-suppressive drugs to more than 100 patients to prevent 1 patient from having a diagnostic endoscopy?

Every clinician involved in the administration of acidsuppressive drugs to critically ill patients should read the observations presented in a recent article in this journal [1]. Krag and colleagues [1] have conducted an international, multi-centre, observational study and provide a modern and relatively precise estimate of the incidence of, and risk factors for, gastrointestinal (GI) bleeding in critically ill patients. Notwithstanding the methodological limitations that the authors acknowledge, these are very important data directly pertaining to clinical practice.

The investigators obtained data from 1034 patients and report that $2.6 \%$ (95\% confidence intervals 1.6, 3.6\%) of patients had an episode of clinically important bleeding. This estimate of incidence is within the historically quoted range of $0.1-4 \%$ [2]. Interestingly, almost $50 \%$ of bleeding episodes were reported in the first 2 days of admission, suggesting that not all bleeds will be amenable to prevention with acid-suppressive prophylaxis commenced in the ICU. Furthermore, some of the bleeding episodes recorded would not be due to 'stress-related mucosal damage' (SRMD), but to separate pathologies, e.g. oesophageal and gastric varices [3]. Previous endoscopy data from critically ill patients who developed clinically significant bleeding indicate that at least onethird of such patients have pathology unrelated to SRMD [4], and given that patients with previous admissions for gastrointestinal bleeding and/or those at high risk of alternative causes of bleeding (e.g. liver cirrhosis) were included, non-SRMD bleeding would have likewise occurred frequently in the study by Krag and colleagues. This is relevant when interpreting these incidence data, as non-SRMD bleeding is also unlikely to be prevented by administration of acid-suppressive drugs.

The term 'clinically significant bleeding' reflects the concept that an episode of bleeding is important to clinicians, but do these bleeding episodes meaningfully impact on patients' ultimate outcomes? Clinicians worry about bleeding because historical studies have reported a strong association with mortality [5, 6]. However, even when acid-suppressive drugs have shown a reduction in episodes of clinically significant bleeding, this has not translated into a reduction in mortality $[5,6]$. These results suggest that bleeding simply identifies patients at greater risk of dying and, when treated, bleeding per se does not cause death [3]. Data from Krag and colleagues 
[1] support this hypothesis: when the investigators conducted an adjusted analysis accounting for possible confounders, bleeding was not associated with mortality.

One reason for the lack of association with mortality is that bleeding may be caused by relatively easy-to-treat pathology. The majority of bleeding episodes in this study were treated only with acid-suppressive drugs (17 patients required only pharmacotherapy and 10 an invasive procedure). Perhaps only bleeding requiring an intervention such as blood transfusion or endoscopy should be considered important, and data from Krag and colleagues suggest that, even if $100 \%$ effective, a preventative strategy would need to be administered to more than 100 patients to prevent one endoscopy.

The study by Krag and colleagues was limited by an inability to collect data related to adverse effects associated with acid-suppressive drugs. Other investigators have reported that acid-suppressive drugs increase the relative risk of pneumonia and $C$. difficile infections up to $50 \%$ and between 50 and $100 \%$, respectively [2, 7-9]. Given that the reported incidence of patients developing ventilator-associated pneumonia is $\sim 10 \%$ and $C$. difficile infections $\sim 3 \%[8,10]$, there is the potential that, for every 100 patients who receive acid-suppressive therapy, an extra patient could develop pneumonia and 1 could develop a $C$. difficile infection. These outcomes are likely to be as important to patients as gastrointestinal bleeding $[10,11]$, and any benefits of prophylaxis would then be counter-balanced or outweighed by these adverse effects.

Despite the potential for harm when prescribing a drug for primary prophylaxis, guidelines frequently include recommendations for the prophylactic administration of acidsuppressive drugs to critically ill patients [12]. This may explain the widespread use of these drugs $[1,13]$. In addition to the considerations outlined regarding the potential adverse effects of these medications, the costs incurred with these frequently prescribed drugs demand that further data are available to inform and guide clinical practice [14].

Only an adequately powered study will determine the impact of the strategy to prophylactically administer these drugs. Because the events (reduction in clinically significant bleeding or increase in pneumonia and $C$. difficile infection) occur infrequently, and very rarely, if ever, directly cause death, an extraordinarily large cohort would need to be studied to determine any effect on mortality.

For these reasons, we suggest that a non-inferiority study focusing on bleeding that is truly important, while also collecting data related to possible adverse effects, is a pragmatic approach to evaluate the merits of empiric prescription of acid-suppressive drugs. Although traditional effectiveness trials ascertain whether a novel treatment is superior to the standard treatment, the noninferiority study is required to evaluate the extent to which withdrawal of a conventional or standard treatment, or its substitution with a novel treatment that is less expensive, will lead to a reduction in benefit [15]. In the proposed non-inferiority study, in which the conventional treatment would be the empiric use of an acid-suppressive drug and the novel treatment placebo, investigators would determine whether the increase in gastrointestinal bleeding with placebo was sufficiently small to warrant withholding prophylaxis (the non-inferiority margin in Fig. 1). Look back at the scenario with which this commentary began: the greater the extent to which prophylaxis increased pneumonia or $C$. difficile infections, the greater the increase in bleeding clinicians would find acceptable. We believe that the comparison of prophylaxis against placebo is ethically justifiable, as clinicians should primum non nocere, and the withdrawal of conventional treatment may only slightly increase the rate of clinically significant bleeding, but this risk is balanced, or even outweighed, by additional episodes of pneumonia, $C$. difficile infection, and the cost resulting from the widespread prophylactic administration of acidsuppressive drugs.

In summary, the study by Krag and colleagues indicate that acid-suppressive drugs continue to be frequently prescribed, but that gastrointestinal bleeding due to SRMD now appears to be an infrequent occurrence. Furthermore, the need for an intervention that is truly important to patients is even less frequent. Based on these observations, the potential benefit from acid-suppressing drugs is likely to be very small. Moreover, prophylaxis is not benign, and may be associated with an increase in adverse events that are at least as important to patients as gastrointestinal bleeding. Resolving this important issue in the management of critically ill patients will, we believe, require an adequately-powered, randomised-controlled, non-inferiority study comparing placebo to an acid-suppressive drug.

Researchers would need to determine the boundaries of primum non nocere, or how much bleeding risk is

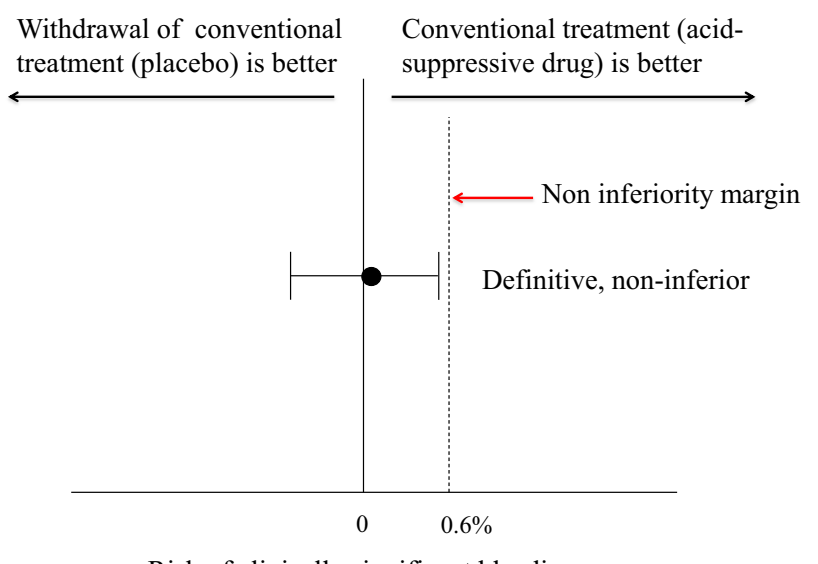

Risk of clinically significant bleeding

Fig. 1 The non-inferiority study design to evaluate the use of empiric acid-suppressive drugs 
tolerable, with the withdrawal of conventional treatment (placebo) when compared to conventional treatment (prophylactic use of an acid-suppressant drug). This would define the non-inferiority margin. If placebo increased the rate of bleeding (both point estimate and $95 \%$ confidence interval) by less than the pre-defined margin (Fig. 1) then withdrawal of conventional treatment is proven non-inferior, which suggests that clinicians should stop the conventional approach to prophylactically administer acid-suppressive drugs (particularly if burden or harm with acid-suppressive drugs is established).

Conflicts of interest On behalf of all authors, the corresponding author states that there is no conflict of interest.

\section{References}

1. Krag M, Perner A, Wetterslev J, Wise MP, Borthwick M, Bendel S, McArthur C, Cook DJ, Nielsen N, Pelosi P et al (2015) Prevalence and outcome of gastrointestinal bleeding and use of acid suppressants in acutely ill adult intensive care patients. Intensive Care Med. doi:10.1007/s00134-015-3725-1

2. Barletta JF, Sclar DA (2014) Proton pump inhibitors increase the risk for hospital-acquired Clostridium difficile infection in critically ill patients. Crit Care 18(6):714

3. Plummer MP, Blaser AR, Deane AM (2014) Stress ulceration: prevalence, pathology and association with adverse outcomes. Crit Care 18(2):213

4. Cook DJ, Fuller HD, Guyatt GH, Marshall JC, Leasa D, Hall R, Winton TL, Rutledge F, Todd TJ, Roy P et al (1994) Risk factors for gastrointestinal bleeding in critically ill patients. Canadian Critical Care Trials Group. N Engl J Med 330(6):377-381

5. Cook D, Guyatt G, Marshall J, Leasa D, Fuller H, Hall R, Peters S, Rutledge F, Griffith L, McLellan A et al (1998) A comparison of sucralfate and ranitidine for the prevention of upper gastrointestinal bleeding in patients requiring mechanical ventilation. Canadian Critical Care Trials Group. N Engl J Med 338(12):791-797
6. Krag M, Perner A, Wetterslev J, Wise MP, Hylander Moller M (2014) Stress ulcer prophylaxis versus placebo or no prophylaxis in critically ill patients. A systematic review of randomised clinical trials with meta-analysis and trial sequential analysis. Intensive Care Med 40(1):11-22

7. MacLaren R, Reynolds PM, Allen RR (2014) Histamine-2 receptor antagonists vs proton pump inhibitors on gastrointestinal tract hemorrhage and infectious complications in the intensive care unit. JAMA Intern Med 174(4):564-574

8. Buendgens L, Bruensing J, Matthes M, Duckers H, Luedde T, Trautwein C, Tacke F, Koch A (2014) Administration of proton pump inhibitors in critically ill medical patients is associated with increased risk of developing Clostridium difficile-associated diarrhea. J Crit Care 29(4):e611-e695

9. Marik PE, Vasu T, Hirani A, Pachinburavan M (2010) Stress ulcer prophylaxis in the new millennium: a systematic review and meta-analysis. Crit Care Med 38(11):2222-2228

10. Nair GB, Niederman MS (2015) Ventilator-associated pneumonia: present understanding and ongoing debates. Intensive Care Med 41(1):34-48
11. Dubberke ER, Olsen MA (2012) Burden of Clostridium difficile on the healthcare system. Clin Infect Dis 55(Suppl 2):S88-S92

12. Dellinger RP, Levy MM, Rhodes A, Annane D, Gerlach H, Opal SM, Sevransky JE, Sprung CL, Douglas IS, Jaeschke R et al (2013) Surviving Sepsis Campaign: international guidelines for management of severe sepsis and septic shock, 2012. Intensive Care Med 39(2):165-228

13. Eastwood GM, Litton E, Bellomo R, Bailey MJ, Festa M, Beasley RW, Young PJ (2014) Opinions and practice of stress ulcer prophylaxis in Australian and New Zealand intensive care units. Crit Care Resusc 16(3):170-174

14. Barletta JF, Sclar DA (2014) Use of proton pump inhibitors for the provision of stress ulcer prophylaxis: clinical and economic consequences. Pharmacoeconomics 32(1):5-13

15. Mulla SM, Scott IA, Jackevicius CA, You JJ, Guyatt GH (2012) How to use a noninferiority trial: users' guides to the medical literature. JAMA 308(24):2605-2611 